\title{
Quantitative tractography reveals changes in the corticospinal tract in drug-naïve children with attention-deficit/hyperactivity disorder
}

\author{
Xuan Bu, PhD*; Chuang Yang, MD*; Kaili Liang, BM; Qingxia Lin, MM; \\ Lu Lu, PhD; Lianqing Zhang, PhD; Hailong Li, PhD; Yingxue Gao, PhD; \\ Shi Tang, MM; Xiaoxiao Hu, MM; Yanlin Wang, PhD; Xinyu Hu, PhD; \\ Meihao Wang, MD; Xiaoqi Huang, PhD, MD
}

\begin{abstract}
Background: The specific role of the corticospinal tract with respect to inattention and impulsive symptoms in children with attentiondeficit/hyperactivity disorder (ADHD) has been explored in the past. However, to our knowledge, no study has identified the exact regions of the corticospinal tract that are affected in ADHD. We aimed to determine comprehensive alterations in the white matter microstructure of the corticospinal tract and underlying neuropsychological substrates in ADHD. Methods: We recruited 38 drug-naïve children with ADHD and 34 typically developing controls. We employed a tract-based quantitative approach to measure diffusion parameters along the trajectory of the corticospinal tract, and we further correlated alterations with attention and response inhibition measures. Results: Compared with controls, children with ADHD demonstrated significantly lower fractional anisotropy and higher radial diffusivity at the level of cerebral peduncle, and higher fractional anisotropy at the level of the posterior limb of the internal capsule in the right corticospinal tract only. As well, increased fractional anisotropy in the posterior limb of the internal capsule was negatively correlated with continuous performance test attention quotients and positively correlated with reaction time on the Stroop Colour-Word Test; increased radial diffusivity in the right peduncle region was positively correlated with omissions in the Stroop test. Limitations: The sample size was relatively small. Moreover, we did not consider the different subtypes of ADHD and lacked sufficient power to analyze subgroup differences. Higher-order diffusion modelling is needed in future white matter studies. Conclusion: We demonstrated specific changes in the right corticospinal tract in children with ADHD. Correlations with measures of attention and response inhibition underscored the functional importance of corticospinal tract disturbance in ADHD.
\end{abstract}

\section{Introduction}

Attention-deficit/hyperactivity disorder (ADHD) is a common neurodevelopmental disorder with an estimated worldwide prevalence of approximately $7.2 \%$ for children younger than 18 years. ${ }^{1}$ Pathophysiological models for ADHD have evolved, from concentrating on frontostriatal-cerebellar circuits to encompassing large-scale distributed networks. ${ }^{2-4}$ Structural connectivity and white matter abnormalities are key elements in these models. Diffusion tensor imaging (DTI) allows for the quantification of white matter microstructure and can reveal the brain substrates of pathologic alterations in structural connectivity. Examining functional abnormalities in key white matter tracts in ADHD can help increase our understanding of the neural mechanisms that underlie ADHD. A meta-analysis of DTI studies in $\mathrm{ADHD}^{5}$ that enrolled mainly children reported white matter abnormalities in the anterior corona radiata and internal capsule, the regions through which the corticospinal tract passes.

Abnormalities related to the corticospinal tract have been reported in ADHD. Using an unbiased, whole-brain, datadriven approach, Hong and colleagues ${ }^{6}$ demonstrated abnormal white matter connectivity linking the postcentral gyrus and cerebellum and significant negative correlations between fractional anisotropy (FA) and continuous performance test (CPT) scores, indicating that white matter connectivity of the corticospinal tract may contribute directly to the poor levels of attention and response inhibition in patients with ADHD.

Correspondence to: X. Huang, Huaxi MR Research Center (HMRRC), Department of Radiology, Sichuan University West China Hospital, 37 Guo Xue Xiang, Chengdu, Sichuan Province 610041, China; julianahuang@163.com

*These authors contributed equally to this work.

Submitted Jan. 29, 2019; Revised May 25, 2019; Accepted Jul. 11, 2019; Published online Nov. 25, 2019

DOI: 10.1503/jpn.190024 
The authors also revealed that the distributed pattern of white matter microstructural integrity separately involving the frontal, striatal and cerebellar regions (some of which can be portions of the corticospinal tract) appears to be disrupted in children with ADHD. In another study, Francx and colleagues ${ }^{7}$ determined that lower FA and higher mean diffusivity in a portion of the corticospinal tract were related to greater remission of hyperactive/impulsive symptoms in patients with ADHD. Given that the corticospinal tract is important in the control of voluntary movements, this study indicated the important role of the corticospinal tract in the hyperactive symptoms of ADHD. Taken together, these results suggest that white matter microstructural abnormalities in the corticospinal tract might underlie the neuropathologic mechanisms of ADHD; thus, more focal white matter abnormalities that reflect neural pathophysiologic mechanisms of ADHD need to be explored.

Although the studies above provided evidence that some symptoms of ADHD might be linked to the integrity of the corticospinal tract, no previous study has focused on a complete description of the abnormalities along its trajectory in ADHD. To determine changes in the white matter microstructure, we assessed the bilateral corticospinal tract by quantifying diffusion parameters at multiple nodes along the tract's trajectory, instead of obtaining the mean value of the whole fibre. Analyzing diffusion measurements along the length of fibre tracts is a powerful approach and can provide much richer information about white matter that may be neglected in mean measures..$^{8-10}$ When there is a change in the mean for a tract, the change might be reflected throughout the entire tract or could be driven principally by a small region within the tract. ${ }^{11}$

In the present study, our goal was to systematically assess white matter microstructure abnormalities in the corticospinal tract using a tract-profile quantification approach, and to explore the associations between disturbed white matter microstructure, clinical symptoms and neuropsychological indexes for attention and response inhibition. This approach allowed us to determine the extent to which white matter microstructural abnormalities in ADHD were localized to focal portions of the corticospinal tract and to further examine the extent to which any focal abnormalities were associated with specific ADHD symptomatology. We used the Integrated Visual and Auditory Continuous Performance Test (IVA-CPT) ${ }^{12}$ and the Stroop Colour-Word Test (Stroop) $)^{13}$ as our neuropsychological tests. The IVA-CPT is one of the most widely used neuropsychological tests in ADHD; it evaluates attention and response control when visual and auditory stimuli are presented. ${ }^{14}$ The Stroop test can measure impaired response inhibition, which is a core impairment in patients with ADHD.

We hypothesized that compared with healthy controls, children with ADHD would show significantly altered FA in focal regions of the corticospinal tract, specifically in the portions of the tract that pass through the brain regions previously shown to be abnormal in ADHD, including the corona radiata, ${ }^{15,16}$ the internal capsule ${ }^{17}$ and the cerebral peduncle. ${ }^{18}$ We further hypothesized that these abnormal- ities would be significantly associated with ADHD symptoms or cognitive deficits.

\section{Methods}

\section{Participants}

A total of 72 children of average intelligence - 38 drug-naïve children with ADHD (age 8.79 $\pm 2.11 \mathrm{yr}$ ) and 34 age-, IQ- and sex-matched typically developing controls (age $9.29 \pm 1.67 \mathrm{yr}$ ) - participated in this study. Children with ADHD were recruited through the Department of Psychiatry, First Affiliated Hospital of Wenzhou Medical University, from June 2013 to December 2017. Children's ADHD diagnosis was confirmed according to the criteria of the Diagnostic and Statistical Manual of Mental Disorders, 5th edition;"19 a semistructured diagnostic interview; and the Schedule for Affective Disorders and Schizophrenia for School-Age Children-Present and Lifetime Version (K-SADS-PL). ${ }^{20}$ Controls were recruited from local primary schools and were screened by the same psychiatrists to confirm the absence of major neurologic or psychiatric disorders, as well as the absence of psychiatric disorders in their first-degree relatives. None of the controls was using any form of psychoactive medication.

Exclusion criteria for both groups included left-handedness, a history of taking psychoactive medications or any other drugs or therapy for disorders, comorbid psychiatric or neurologic disorders, traumatic brain injury and a history of psychiatric conditions in first-degree relatives. All participants were right-handed and required to show a full scale IQ above 90 on the Wechsler Intelligence Scale for ChildrenChinese Revision. ${ }^{21}$

The study was approved by the ethics committee of the First Affiliated Hospital of Wenzhou Medical University. All participants and their parents were fully informed about the purpose and procedures of the study, and written informed consent was obtained from the parents.

\section{Behavioural assessment and neuropsychological measures}

To measure the behavioural problems of patients with ADHD, we used the Chinese version of the revised Conners' Parent Rating Scale (CPRS), ${ }^{22}$ which has good reliability and validity among Chinese children with ADHD. ${ }^{23}$ The CPRS is a battery of questions used to evaluate problematic behaviour across areas such as sleep, temper and peer relationships. The parents rate each behaviour on a 4-point Likert scale. The behaviours are aggregated into the following groups: conduct problems, study problems, psychosomatic problems, impulsive-hyperactive behaviour, anxiety and hyperactivity index. Higher scores indicate more severe problems.

Response inhibition was evaluated using a Chinese modified version of the computerized Stroop Colour-Word Test. ${ }^{13}$ Participants were presented with a series of stimuli (colour words or squares) printed in red or blue (with the word corresponding to or contradicting the colour at random) and asked to click the left button when they saw red and the right 
button when they saw blue. They were instructed that their responses should be as quick and as accurate as possible. Each stimulus remained on the screen for $1000 \mathrm{~ms}$, or until a response was collected. If no response was collected within $1000 \mathrm{~ms}$, or the participant's response was incorrect, that response was scored as a missing or incorrect, respectively. Software recorded the time between each stimulus onset and the participant's response, and it automatically calculated total reaction time and the number of correct, incorrect and missing responses. The entire test took $90 \mathrm{~s}$, less time than required for the traditional paper version of the Stroop test.

The IVA-CPT ${ }^{12}$ evaluates attention and response control at the auditory and visual levels. The test provides 6 global indexes that aim to summarize the overall performance of participants in terms of response control and attention in both visual and auditory dimensions.

\section{MRI data acquisition and preprocessing}

We performed whole-brain MRI using a GE signal HDx $3 \mathrm{~T}$ MRI scanner with an 8-channel phase-array head coil. Sequences included a spoiled gradient-recalled echo sequence to acquire $T_{1}$-weighted images (repetition time $7200 \mathrm{~ms}$, echo time $2.2 \mathrm{~ms}, 176$ axial slices, slice thickness $1 \mathrm{~mm}$, flip angle $7^{\circ}$, matrix size $256 \times 256$, field of view $256 \mathrm{~mm}$ ) and a single-shot echo-planar sequence (repetition time $8875 \mathrm{~ms}$, echo time $87.9 \mathrm{~ms}, 33$ axial-oblique slices, slice thickness $4 \mathrm{~mm}$, slice gap $1 \mathrm{~mm}$, matrix size $130 \times 128$, field of view $240 \mathrm{~mm})$ with 30 directions $\left(b=900 \mathrm{~s} / \mathrm{mm}^{2}\right)$ and 1 unweighted $b 0$ scan $\left(b=0 \mathrm{~s} / \mathrm{mm}^{2}\right)$.

We conducted eddy current correction, motion distortion and skull stripping using the FMRIB version 5.0 (Oxford Centre for Functional MRI of the Brain, www.fmrib.ox. ac.uk/fsl/) diffusion toolbox (FDT) and brain extraction tool. A diffusion tensor model was fitted at each voxel using the FDT, generating eigenvalues and eigenvectors that we used to calculate FA, mean diffusivity, axial diffusivity and radial diffusivity. We applied the script dtiMakeDt6FromFSL from the MrDiffusion toolkit (http:/ / white.stanford.edu/newlm/ index.php/MrDiffusion) to align the $T_{1}$ image to the $\mathrm{S} 0 \mathrm{im}$ age to obtain a dt6 file for further analysis.

\section{Automated fibre quantification}

We used automated fibre quantification (AFQ; github.com/ jyeatman/AFQ) to identify and characterize diffusion parameters along the bilateral corticospinal tract. A detailed description of this approach is provided in a previous study. ${ }^{24}$ The identification procedures included the following primary steps: (1) whole-brain deterministic tractography was conducted with an FA threshold of 0.2 and an angle threshold of $30^{\circ}, 25$ (2) streamlines that passed through 2 waypoint regions of interest were identified as candidate corticospinal tract fibres ( 1 region of interest defined the entire cerebral peduncle in an axial plane at the level of the decussation of the superior cerebellar peduncle, and the other was placed in the central sulcus right after the bifurcation to the motor and sensory cortex); ${ }^{26}$ (3) in probability-map-based fibre refinement, streamlines with lengths longer than 5 standard deviations above the mean or Mahalanobis distances greater than 4 standard deviations from the corticospinal tract spatial core were removed, ${ }^{27}$ (4) each fibre was sampled at 100 equidistant nodes that could be used to compute diffusion parameters and tract volume along the fibre.

\section{Statistical analyses}

We investigated group differences in demographic characteristics using 2-sample $t$ tests or $\chi^{2}$ tests. To compare the diffusion parameters between patients with ADHD and controls, we plotted the values and standard error of the mean for each point and conducted $t$ tests point-wise along each tract for 100 nodes. To explore the relationship between diffusion parameters and clinical measurements in patients with ADHD, we performed Pearson correlation analysis for point-wise data (extracted from significant nodes between groups) between diffusion parameters and clinical assessment scores for ADHD and controls separately. Significance was set at $p=0.05$ for all analyses, and family-wise error (FWE) correction was applied to adjust for multiple comparisons. The FWE correction used in the present study was an implementation of the permutation method described by Nichols and Holmes. ${ }^{28}$

\section{Results}

\section{Demographic and clinical characteristics}

The ADHD and control groups did not differ significantly in terms of age, sex or IQ. The groups did differ significantly with respect to CPRS score. As expected, patients with ADHD had more severe behaviour and study problems and were more hyperactive and impulsive. In the Stroop Colour-Word Test, performance in the ADHD group was also poorer, including fewer correct numbers $(p=0.018)$ and longer reaction times $(p=0.008)$. In the IVACPT, patients with ADHD exhibited lower means for all indexes, but we observed significant differences only in the attention indexes $(p<0.001)$. Sample characteristics are summarized in Table 1.

\section{Group differences in diffusion properties and correlation analysis}

We examined differences between the 2 groups in terms of diffusion properties and volume at each node along the bilateral corticospinal tract. For the right corticospinal tract, we found significantly lower FA in patients with ADHD than in controls on nodes 16 to 24 , at the level of cerebral peduncle, and higher FA on nodes 83 to 95, at the level of the posterior limb of the internal capsule (PLIC). Patients with ADHD also displayed significantly higher radial diffusivity at the level of the cerebral peduncle. We found no group differences for the left corticospinal tract, bilateral tract averaged value or volume. The diffusion properties of the corticospinal tract are shown in Figure 1. 
Table 1: Demographic characteristics, behavioural measures and executive function in patients with ADHD and controls

\begin{tabular}{|c|c|c|c|c|}
\hline \multirow[b]{2}{*}{ Characteristic } & \multicolumn{2}{|c|}{ Group, mean \pm SD } & \multicolumn{2}{|c|}{ Statistics } \\
\hline & $\operatorname{ADHD}(n=38)$ & Controls $(n=34)$ & $t / \chi^{2}$ & $p$ value \\
\hline Age, yr & $8.79 \pm 2.11$ & $9.29 \pm 1.67$ & $t_{70}=-1.12$ & 0.27 \\
\hline Sex, male/female & $29 / 9$ & $21 / 13$ & $\chi^{2}{ }_{1}=1.79$ & 0.18 \\
\hline IQ score & $120.63 \pm 16.34$ & $122.18 \pm 13.58$ & $t_{70}=-0.43$ & 0.67 \\
\hline \multicolumn{5}{|l|}{ Conners Parent Rating Scale } \\
\hline Conduct problem & $1.11 \pm 0.55$ & $0.37 \pm 0.32$ & $t_{61}=7.03$ & $<0.001$ \\
\hline Psychosomatic & $0.44 \pm 0.34$ & $0.12 \pm 0.21$ & $t_{63}=4.71$ & $<0.001$ \\
\hline Anxiety & $0.61 \pm 0.44$ & $0.32 \pm 0.29$ & $t_{65}=3.23$ & 0.002 \\
\hline Study problem & $1.95 \pm 0.61$ & $0.58 \pm 0.55$ & $t_{70}=9.94$ & $<0.001$ \\
\hline Hyperactivity-impulsivity & $1.50 \pm 0.72$ & $0.50 \pm 0.50$ & $t_{66}=6.88$ & $<0.001$ \\
\hline Hyperactivity index & $1.46 \pm 0.54$ & $0.46 \pm 0.39$ & $t_{70}=8.93$ & $<0.001$ \\
\hline \multicolumn{5}{|l|}{ Stroop Colour-Word Test } \\
\hline Total time, ms & $3101.58 \pm 789.23$ & $2433.29 \pm 1307.40$ & $t_{53}=2.59$ & 0.012 \\
\hline Correct answers, $n$ & $42.76 \pm 17.60$ & $52.91 \pm 20.41$ & $t_{70}=-2.27$ & 0.027 \\
\hline Errors, $n$ & $35.08 \pm 10.57$ & $32.35 \pm 11.29$ & $t_{70}=1.06$ & 0.29 \\
\hline Missing, $n$ & $43.03 \pm 23.41$ & $35.59 \pm 21.70$ & $t_{70}=1.39$ & 0.17 \\
\hline \multicolumn{5}{|l|}{ Continuous performance test } \\
\hline Full scale control quotient & $73.16 \pm 27.32$ & $79.68 \pm 21.57$ & $t_{70}=-1.16$ & 0.27 \\
\hline Visual control quotient & $75.37 \pm 27.77$ & $82.76 \pm 21.10$ & $t_{70}=-1.26$ & 0.21 \\
\hline Auditory control quotient & $74.21 \pm 28.88$ & $80.05 \pm 21.95$ & $t_{70}=-1.09$ & 0.28 \\
\hline Full scale attention quotient & $72.32 \pm 24.76$ & $95.53 \pm 20.46$ & $t_{70}=-4.31$ & $<0.001$ \\
\hline Visual attention quotient & $75.37 \pm 23.29$ & $94.65 \pm 18.64$ & $t_{70}=-3.85$ & $<0.001$ \\
\hline Auditory attention quotient & $71.21 \pm 29.37$ & $95.79 \pm 20.89$ & $t_{70}=-4.05$ & $<0.001$ \\
\hline
\end{tabular}

To explore the white matter microstructural implications of group differences in FA and radial diffusivity, we correlated FA and radial diffusivity with participants' scores on the Stroop test and IVA-CPT. In patients with ADHD, increased FA was negatively correlated with 3 attention quotients in the IVA-CTP (full scale attention quotient, auditory attention quotient and visual attention quotient) and positively correlated with reaction time in the Stroop test. Increased radial diffusivity in the ADHD group was positively correlated with the number of omissions in the Stroop test. We found no significant correlations in the control group. Correlation details are shown in Figure 2.

Removing 1 outlier from the ADHD group did not substantially alter the original results; the same segment of the right corticospinal tract still showed significant alterations and a significant relationship between altered diffusion parameters and psychological measurements in the same direction.

\section{Discussion}

In this study of children with ADHD versus controls, the findings demonstrated regional abnormalities in the microstructure of the right corticospinal tract in children with ADHD. Decreased FA and increased radial diffusivity at the level of the cerebral peduncle may be related to worse response inhibition in ADHD, and increased FA at the level of the internal capsule may be associated with disrupted attention. Taken together, these results revealed that white matter microstructural deficits in the right corticospinal tract are specific to certain segments, and that these abnormalities correspond to different types of white matter pathology at a neurobiological level. These findings suggest that the corticospinal tract is associated with the neural underpinnings of inattention and executive dysfunction in children with ADHD.

\section{Alterations in white matter microstructural properties in the PLIC}

In line with earlier DTI studies ${ }^{7,29}$ that showed increased FA in the superior part of the corticospinal tract in children with ADHD, we found that the superior part of the corticospinal tract, which is at the level of the PLIC, presented significantly higher FA in children with ADHD than in controls. Diffusion tensor imaging studies in ADHD commonly attribute increased FA to reduced fibre branching ${ }^{30,31}$ or excessive myelin hyperplasia resulting from an adaptive process in response to worsening cognitive and executive function in ADHD. ${ }^{32} \mathrm{Be}-$ cause we found a positive association between higher FA and longer reaction times on the Stroop test, it is reasonable to infer that decreases in inhibitory responses may correspond to ongoing myelination of the corticospinal tract. As well, in areas with less fibre branching, FA may be relatively high if the magnitude of diffusion across secondary and tertiary directions is reduced. ${ }^{33}$ However, the lack of a significant decrease in radial diffusivity in our study did not support reduced branching of the right corticospinal tract at the level of the PLIC. Future research using more advanced imaging methods that allow for higher resolution of crossing fibres 
A

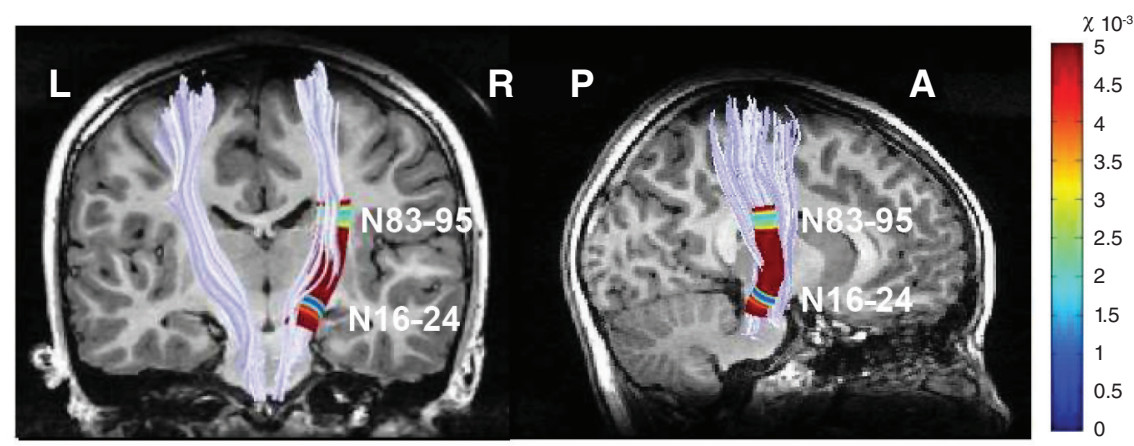

B
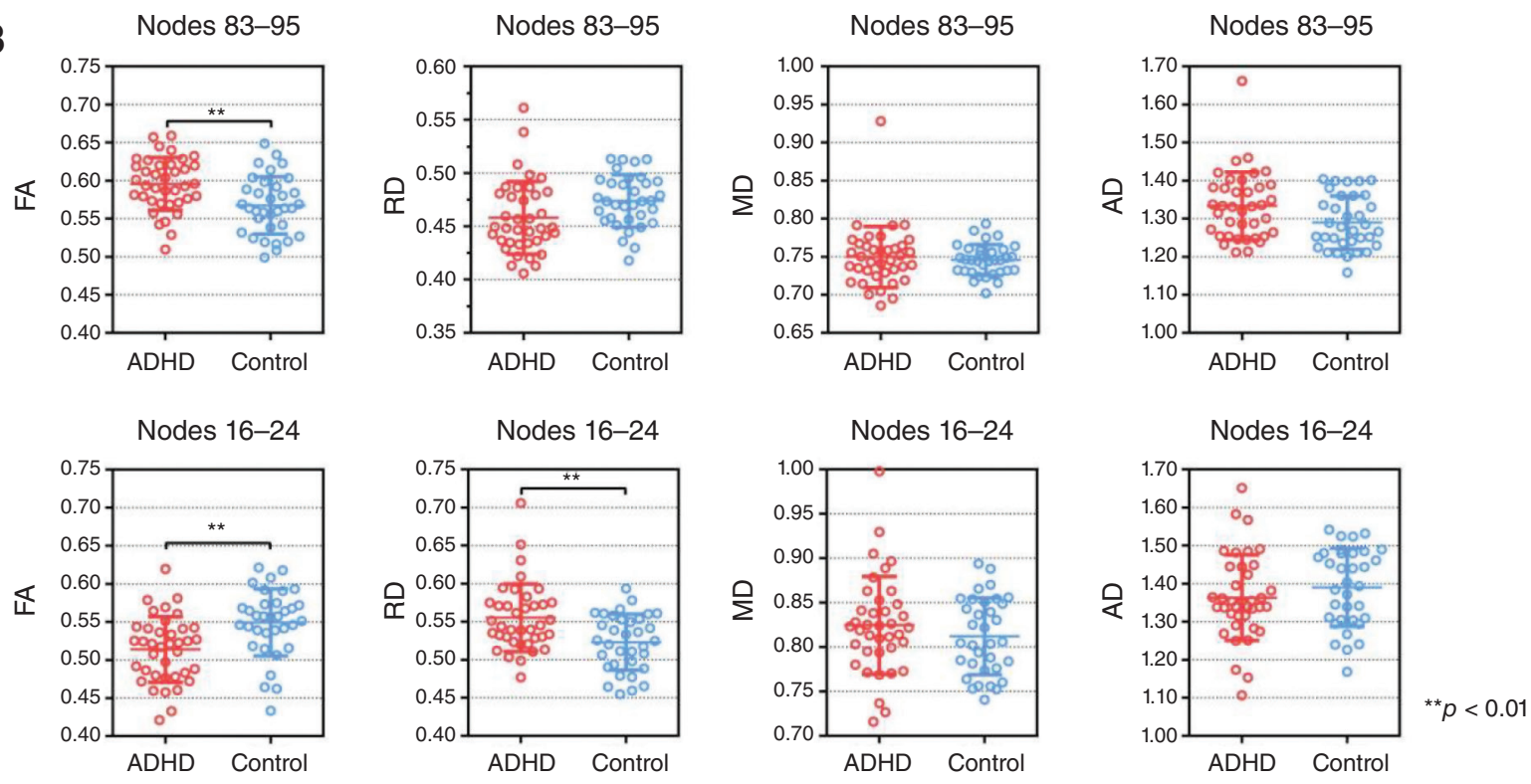

Fig. 1: Automated fibre tract quantification - corticospinal tracts and group differences in fractional anisotropy (FA), radial diffusivity (RD), mean diffusivity (MD) and axial diffusivity (AD). (A) Right corticospinal tract with significant group differences ( $p$ values are associated with the colours of a heat map; $p_{\mathrm{FWE}}<0.05$ ). (B) Scatter plots for FA, RD, MD and AD in 2 significant regions of the right corticospinal tract. Error bars represent standard deviation. Asterisks indicate statistical significance from 2-sample $t$ tests between groups. ADHD = attention-deficit/ hyperactivity disorder FWE = family-wise error.

may permit a better assessment of whether increases in corticospinal tract FA in ADHD are related to abnormalities in the regions of crossing fibres.

A novel finding of the present study was the negative association between FA in the right PLIC and attention quotient in children with ADHD, which demonstrated that the right corticospinal tract (rather than the most studied frontostriatal tracts) was associated with inattention symptoms in children with ADHD. Greater FA values may be associated with a higher level of ADHD symptomatology. ${ }^{7,34}$ Our findings were compatible with those of a tractography-based study by Hong and colleagues, ${ }^{6}$ who found a negative relationship between FA in the white matter connectivity from the right postcentral gyrus to the cerebellar hemisphere and CPT omission errors (a measure of inattention) and response time variability (a measure of the consistency of attention) in chil- dren with ADHD. The right hemisphere plays a key role in selective attention, arousal and vigilance, ${ }^{35}$ as well, based on previous studies, ${ }^{36,37}$ ADHD may involve abnormal right hemisphere processing. Thus, right hemispheric attentional disturbance may exist in people with ADHD. This idea is supported by our results demonstrating that regional white matter abnormalities were localized to the right corticospinal tract among children with ADHD and associated with deficits in attentional processes, as observed through their performance in response to visual and auditory stimuli.

\section{Alterations in white matter microstructural properties in the cerebral peduncle}

Consistent with previous studies, ${ }^{33,38-40}$ we found lower FA in the corticospinal tract in children with ADHD. We also found 
A

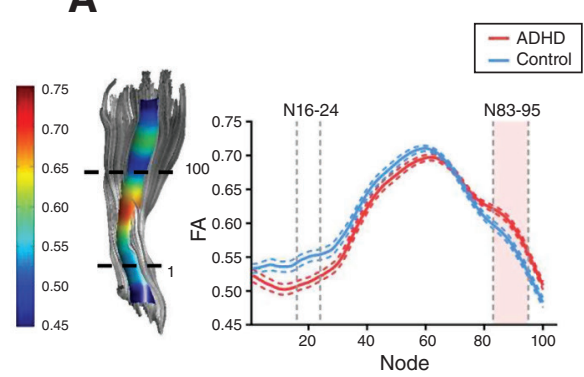

C
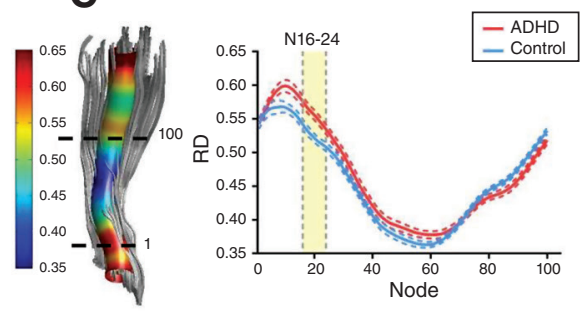

B

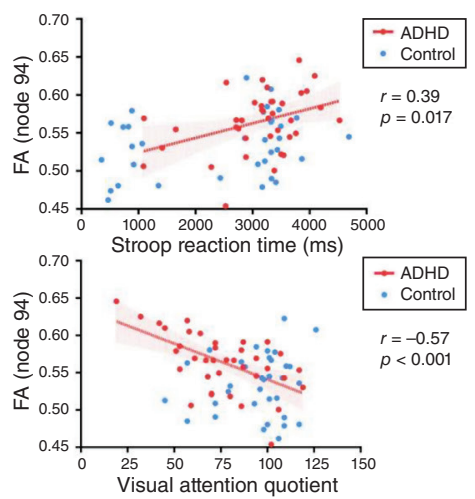

D

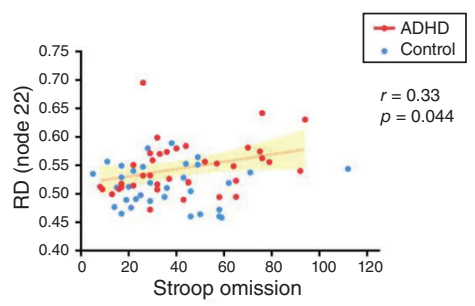

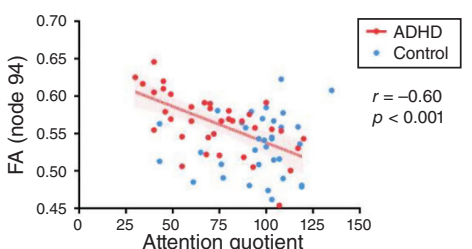

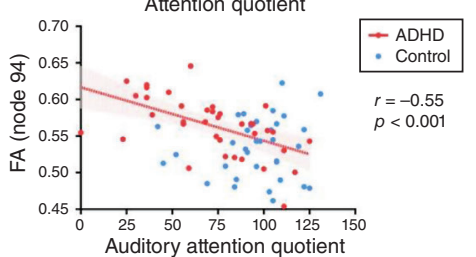

Auditory attention quotient

Fig. 2: $(A, C)$ Diffusion profiles between nodes 1 and 100 for the right corticospinal tract in children with attention-deficit/hyperactivity disor$\operatorname{der}(A D H D)$ and typically developing controls. The 3D rendering derived from automated fibre tract quantification software is shown for a single representative participant with ADHD. The defining regions of interest are depicted as dotted lines (fractional anisotropy [FA] values are associated with the colours of a heat map). Adjacent to the rendering, FA/radial diffusivity (RD) profiles show the FA/RD at 100 equidistant nodes along the corticospinal tract between the defining regions of interest. Solid lines represent the mean FA/RD, and dotted lines denote the standard error of the mean. Red lines: children with ADHD. Blue lines: typically developing controls. Nodes that showed significant differences are marked on the 3D-rendered tract ( $p$-values are associated with the colours of a heat map, $\left.p_{\mathrm{FWE}}<0.05\right)$. (B,D) Correlation between neuropsychological measurement and diffusion parameters for the right corticospinal tract. Representative node 94 is plotted for FA, and representative node 22 is plotted for $\mathrm{RD}$. Red lines represent the linear correlation between neuropsychological measurement and diffusion parameters in children with $A D H D$, and the coloured area indicates the $95 \%$ confidence intervals FWE $=$ family-wise error.

higher radial diffusivity and lower FA in the overlapping area in the right corticospinal tract. Fractional anisotropy, an indicator of white matter coherence and axonal organization, usually decreases in damaged, disorganized or atrophied white matter. ${ }^{41}$ Radial diffusivity represents perpendicular diffusion of water; higher values are thought to reflect decreased myelination. ${ }^{42}$ Given that previous studies in young children with ADHD suggested reduced or delayed myelination, ${ }^{43}$ our findings suggest that impaired myelination may contribute to corticospinal tract pathophysiology at the level of the cerebral peduncle.

Most previous studies did not emphasize specific regions of the corticospinal tract except one, which indicated lower FA in the right cerebral peduncle. ${ }^{38}$ The cerebral peduncle assists in refining motor movements, learning new motor skills and converting proprioceptive information into balance and posture maintenance. ${ }^{44,45}$ Alterations in this region might contribute to motor deficits in children with ADHD. Our results were derived from the AFQ method, which graphically tracked the course of the corticospinal tract and computed FA at multiple equidistant nodes with more precise spatial localizations, providing greater evidence for the existence of disrupted white matter microstructure in the corticospinal tract at the level of the cerebral peduncle.

In the present study, the positive correlation between impaired white matter microstructure at the cerebral peduncle and the number of omissions in the Stroop test among children with ADHD added some evidence to support the concept that white matter microstructure of the corticospinal tract may be essential for response inhibition in ADHD. One of the core cognitive and neuropsychological characteristics of people with ADHD is problems with response inhibition. ${ }^{46}$ Some scholars have indicated that energetic failures underlie the inhibitory dysfunction in children with ADHD according to the cognitive-energetic model. This model approaches inhibitory deficiency in ADHD through 3 levels: motor organization as the output side at the first level is directly related to the activation pool at the second level, and that is especially relevant to response inhibition, which is part of executive function at the third level. ${ }^{47}$ Together with previous studies, our work demonstrates that motor deficits related to aberrations in 
the white matter microstructure of the cerebral peduncle may play a role in inhibition failures.

\section{Limitations}

Our study had several limitations. First, given our relatively small sample size, we calculated effect size and found that the effect size for significant comparisons was greater than 0.35 , indicating that our statistical power was fair and valid (see details on effect size in Appendix 1, available at jpn. ca/190024-a1). Second, we did not consider the different subtypes of ADHD, and we lacked sufficient power to analyze subgroup differences. Further analyses of subtype differences may be informative. Third, given the incidence of motor difficulties in ADHD and our focus on the corticospinal tract, which is primarily a motor tract, we should have performed motor skill assessment during participant recruitment to conduct group comparisons for motor skill measures, or used these measures as covariates. However, the children with ADHD who enrolled in this study had no comorbidities related to motor difficulties, such as developmental coordination disorder or Tourette syndrome; we suggest that motor skills deficits had little effect on our results. Fourth, we admit that the $b$ value in the present study was relatively low, which may have led to less sensitivity to the orientation of fibres and affected the quantitation of diffusion parameters. Nonetheless, we reconstructed corticospinal tracts that were well documented in previous anatomic studies using regions of interest based on a priori knowledge, and we visually checked the fibre pathway for all participants to ensure precision and accuracy in the tracts. Fifth, the tensor model we used was limited in areas with multiple fibre populations, because only 1 fibre direction per voxel was modelled. Advanced acquisition techniques, such as high angular resolution diffusion imaging or diffusion spectrum imaging, will enable more complex models to better reflect multiple fibre orientations. For example, previous studies have highlighted the benefits of higher-order diffusion MRI (e.g., constrained spherical deconvolution) relative to DTI for clarifying earlier inconsistencies in terms of the profile of corticospinal tract organization in developmental coordination disorder ${ }^{48}$ and to characterize frontostriatal white matter properties and topological organization in ADHD. ${ }^{49,50}$ Future work can try to move beyond the DTI framework to higher-order diffusion modelling of white matter organization to comprehensively characterize microstructure in ADHD.

\section{Conclusion}

The present study revealed characteristic white matter alterations along the right corticospinal tract in children with ADHD. Alterations at the level of the cerebral peduncle were characterized by lower FA and higher radial diffusivity, which were related to worse response inhibition. In contrast, at the level of the internal capsule, the right corticospinal tract showed higher FA, and this appeared to be more closely linked to inattention. This study provided evidence that the right corticospinal tract at the level of the PLIC and cerebral peduncle is involved in the neuropathological underpinnings of ADHD. We also highlighted the benefit of adopting quantitative tract-oriented tractography to improve power to detect effects, and we suggest that such an approach provides improved sensitivity.

Acknowledgments: This study was supported by the National Natural Science Foundation (Grant No. 81671669) and Science and Technology Project of Sichuan Province (Grant No. 2017JQ0001).

Affiliations: From the Huaxi MR Research Center (HMRRC), Department of Radiology, Sichuan University West China Hospital, 37 Guo Xue Xiang, Chengdu, Sichuan 610041, China (Bu, Liang, $\mathrm{Lu}$, Zhang, Li, Gao, Tang, Hu, Wang, Hu, Huang); the Department of Psychiatry, Wenzhou Medical University First Affiliated Hospital, Nan Bai Xiang, Wenzhou, Zhejiang 325003, China (Yang, Lin); and the Department of Radiology, Wenzhou Medical University First Affiliated Hospital, Nan Bai Xiang, Wenzhou, Zhejiang 325003, China (Wang).

\section{Competing interests: None declared.}

Contributors: X. Bu, C. Yang, and X. Huang designed the study. Q. Lin and C. Yang acquired the data, which all authors analyzed. X. Bu, C. Yang, and X. Huang, which all authors reviewed. All authors approved the final version to be published and can certify that no other individuals not listed as authors have made substantial contributions to the paper.

\section{References}

1. Thomas R, Sanders S, Doust J, et al. Prevalence of attention-deficit/ hyperactivity disorder: a systematic review and meta-analysis. Pediatrics 2015;135:e994-1001.

2. Castellanos FX, Proal E. Large-scale brain systems in ADHD: beyond the prefrontal-striatal model. Trends Cogn Sci 2012;16:17-26.

3. Liston C, Malter Cohen M, Teslovich T, et al. Atypical prefrontal connectivity in attention-deficit/hyperactivity disorder: pathway to disease or pathological end point? Biol Psychiatry 2011. 69:1168-77.

4. Konrad K, Eickhoff SB. Is the ADHD brain wired differently? A review on structural and functional connectivity in attention deficit hyperactivity disorder. Hum Brain Mapp 2010;31:904-16.

5. van Ewijk H, Heslenfeld DJ, Zwiers MP, et al. Diffusion tensor imaging in attention deficit/hyperactivity disorder: a systematic review and meta-analysis. Neurosci Biobehav Rev 2012;36:1093-106.

6. Hong SB, Zalesky A, Fornito A, et al. Connectomic disturbances in attention-deficit/hyperactivity disorder: a whole-brain tractography analysis. Biol Psychiatry 2014;76:656-63.

7. Francx $W$, Zwiers MP, Mennes M, et al. White matter microstructure and developmental improvement of hyperactive/impulsive symptoms in attention-deficit/hyperactivity disorder. J Child Psychol Psychiatry 2015;56:1289-97.

8. Wang Y, Olson IR. The original social network: white matter and social cognition. Trends Cogn Sci 2018;22:504-16.

9. Yeatman JD, Dougherty RF, Myall NJ, et al. Tract profiles of white matter properties: automating fiber-tract quantification. PLoS One 2012;7:e49790

10. Jbabdi S, Johansen-Berg H. Tractography: where do we go from here? Brain Connect 2011;1:169-83.

11. Tsang JM, Dougherty RF, Wandell BA. Tract alignment errors decrease detection power in group analyses of diffusion data with TBSS. Society for Neuroscience: San Diego (CA); 2010.

12. Tinius TP. The Integrated Visual and Auditory Continuous Performance Test as a neuropsychological measure. Arch Clin Neuropsychol 2003;18:439-54.

13. Pilli R, Naidu M, Pingali UR, et al. A computerized Stroop test for the evaluation of psychotropic drugs in healthy participants. Indian J Psychol Med 2013;35:180-9.

14. Frazier TW, Demaree Ha, Youngstrom EA. Meta-analysis of intellectual and neuropsychological test performance in attention-deficit/ hyperactivity disorder. Neuropsychology 2004;18:543-55. 
15. Nagel BJ, Bathula D, Herting M, et al. Altered white matter microstructure in children with attention-deficit/hyperactivity disorder. I Am Acad Child Adolesc Psychiatry 2011;50:283-92.

16. Cortese S, Imperati D, Zhou J, et al. White matter alterations at 33year follow-up in adults with childhood attention-deficit/hyperactivity disorder. Biol Psychiatry 2013;74:591-8.

17. Pavuluri MN, Yang S, Kamineni K, et al. Diffusion tensor imaging study of white matter fiber tracts in pediatric bipolar disorder and attention-deficit/hyperactivity disorder. Biol Psychiatry 2009;65:586-93.

18. Ashtari M, Kumra S, Bhaskar SL, et al. Attention-deficit/hyperactivity disorder: a preliminary diffusion tensor imaging study. Biol Psychiatry 2005;57:448-55.

19. American Psychiatric Association. Diagnostic and statistical manual of mental disorders. 5th ed. Washington (DC): American Psychiatric Association; 2013.

20. Kaufman J, Birmaher B, Brent DA, et al. K-SADS-PL. I Am Acad Child Adolesc Psychiatry. 2000;39(10):1208.

21. Gong YX, Cai TS. The test analysis of China-Wechsler young children scale of intelligence. Chinese J Clin Psychol 1994;1:1-6.

22. Conners CK, Sitarenios G, Parker JD, et al. The revised Conners' Parent Rating Scale (CPRS-R): factor structure, reliability, and criterion validity. J Abnorm Child Psychol 1998;26:257-68.

23. Su L, Li X, Huang C, et al. Norms of the Conners parent symptom questionnaire in Chinese urban children. Chin J Clin Psychol 2001; 9:241-3.

24. Yeatman JD, Dougherty RF, Myall NJ, et al. Tract profiles of white matter properties: automating fiber-tract quantification. PLoS One 2012;7:e49790.

25. Basser PJ, Pajevic S, Pierpaoli C, et al. In vivo fiber tractography using DT-MRI data. Magn Reson Med 2000;44:625-32.

26. Wakana S, Caprihan A, Panzenboeck MM, et al. Reproducibility of quantitative tractography methods applied to cerebral white matter. Neuroimage 2007;36:630-44.

27. Johnson RT, Yeatman JD, Wandell BA, et al. Diffusion properties of major white matter tracts in young, typically developing children. Neuroimage 2014;88:143-54.

28. Nichols TE, Holmes AP. Nonparametric permutation tests for functional neuroimaging: a primer with examples. Hum Brain Mapp 2002;15:1-25.

29. Svatkova A, Nestrasil I, Rudser K, et al. Unique white matter microstructural patterns in ADHD presentations - a diffusion tensor imaging study. Hum Brain Mapp 2016;37:3323-36.

30. Mori S, Zhang J. Principles of diffusion tensor imaging and its applications to basic neuroscience research. Neuron 2006;51:527-39.

31. Silk TJ, Vance A, Rinehart N, et al. White-matter abnormalities in attention deficit hyperactivity disorder: a diffusion tensor imaging study. Hum Brain Mapp 2009;30:2757-65.

32. Li Q, Sun J, Guo L, et al. Increased fractional anisotropy in white matter of the right frontal region in children with attention-deficit/ hyperactivity disorder: a diffusion tensor imaging study. Neuroendocrinol Lett 2010;31:747-53.

33. Suzuki Y, Matsuzawa H, Kwee IL, et al. Absolute eigenvalue diffusion tensor analysis for human brain maturation. NMR Biomed 2003; 16:257-60.
34. Van Ewijk H, Heslenfeld DJ, Zwiers MP, et al. Different mechanisms of white matter abnormalities in attention-deficit/hyperactivity disorder: a diffusion tensor imaging study. I Am Acad Child Adolesc Psychiatry 2014;53:790-9, e3.

35. Pardo JV, Fox PT, Raichle ME. Localization of a human system for sustained attention by positron emission tomography. Nature 1991;349:61-4

36. Sheppard DM, Bradshaw JL, Mattingley JB, et al. Effects of stimulant medication on the lateralisation of line bisection judgements of children with attention deficit hyperactivity disorder. J Neurol Neurosurg Psychiatry 1999;66:57-63.

37. Zou H, Yang J. Exploring the brain lateralization in ADHD based on variability of resting-state fMRI signal. J Atten Disord 2018 ; 1087054718816170

38. Ashtari M, Kumra S, Bhaskar SL, et al. Attention-deficit/hyperactivity disorder: a preliminary diffusion tensor imaging study. Biol Psychiatry 2005;57:448-55

39. Hamilton LS, Levitt JG, O'Neill J, et al. Reduced white matter integrity in attention-deficit hyperactivity disorder. Neuroreport 2008 19:1705-8.

40. Ameis SH, Lerch JP, Taylor MJ, et al. A diffusion tensor imaging study in children with ADHD, autism spectrum disorder, OCD, and matched controls: distinct and non-distinct white matter disruption and dimensional brain-behavior relationships. Am J Psychiatry 2016;173:1213-22.

41. Thomason ME, Thompson PM. Diffusion imaging, white matter, and psychopathology. Annu Rev Clin Psychol 2011;7:63-85.

42. Song SK, Sun SW, Ramsbottom MJ, et al. Dysmyelination revealed through MRI as increased radial (but unchanged axial) diffusion of water. Neuroimage 2002;17:1429-36.

43. Nagel BJ, Bathula D, Herting M, et al. Altered white matter microstructure in children with attention-deficit/hyperactivity disorder. J Am Acad Child Adolesc Psychiatry 2011;50:283-92.

44. Hendelman W. Atlas of functional neuroanatomy. 3rd ed. Boca Raton (FL): CRC Press; 2006.

45. Swenson RS. Cerebellar systems. In: Swenson RS. Review of clinical and functional neuroscience. Hanover (NH): Dartmouth Medical School; 2006.

46. Hinshaw SP. Attention deficit hyperactivity disorder (ADHD): controversy, developmental mechanisms, and multiple levels of analysis. Annu Rev Clin Psychol 2018;14:291-316.

47. Sergeant J. The cognitive-energetic model: an empirical approach to attention-deficit hyperactivity disorder. Neurosci Biobehav Rev 2000;24:7-12.

48. Hyde C, Fuelscher I, Enticott PG, et al. White matter organization in developmental coordination disorder: a pilot study exploring the added value of constrained spherical deconvolution. Neuroimage Clin 2019;21:101625

49. Silk TJ, Vilgis V, Adamson C, et al. Abnormal asymmetry in frontostriatal white matter in children with attention deficit hyperactivity disorder. Brain Imaging Behav 2016;10:1080-9.

50. Beare R, Adamson C, Bellgrove MA, et al. Altered structural connectivity in ADHD: a network based analysis. Brain Imaging Behav 2017;11:846-58. 